\title{
Radiation emitted by transverse-gradient undulators
}

\author{
Axel Bernhard, Nils Braun, Verónica Afonso Rodríguez, Peter Peiffer, \\ Robert Rossmanith, and Christina Widmann \\ Karlsruhe Institute of Technology, Laboratory for Applications of Synchrotron Radiation, \\ Kaiserstrasse 12, 76131 Karlsruhe, Germany \\ Michael Scheer \\ Helmholtz-Zentrum Berlin, Albert-Einstein-Strasse 15, 12489 Berlin, Germany
}

(Received 5 April 2016; published 20 September 2016)

\begin{abstract}
Conventional undulators are used in synchrotron light sources to produce radiation with a narrow relative spectral width as compared to bending magnets or wigglers. The spectral width of the radiation produced by conventional undulators is determined by the number of undulator periods and by the energy spread and emittance of the electron beam. In more compact electron sources like for instance laser plasma accelerators the energy spread becomes the dominating factor. Due to this effect these electron sources cannot in general be used for high-gain free electron lasers (FELs). In order to overcome this limitation, modified undulator schemes, so-called transverse gradient undulators (TGUs), were proposed and a first superconducting TGU was built at Karlsruhe Institute of Technology (KIT), Karlsruhe, Germany. In this paper simulations of the expected synchrotron radiation spectral distribution are presented. An experimental test with that device is under preparation at the laser wakefield accelerator at the JETI laser at the University of Jena, Germany.
\end{abstract}

DOI: 10.1103/PhysRevAccelBeams.19.090704

\section{INTRODUCTION}

In a conventional undulator the fundamental wavelength $\lambda$ of the emitted radiation is given by

$$
\lambda=\frac{\lambda_{\mathrm{u}}}{2 \gamma^{2}}\left(1+\frac{K^{2}}{2}\right)
$$

$\lambda_{\mathrm{u}}$ is the period length of the undulator, $K=\frac{e}{2 \pi m c} \lambda_{\mathrm{u}} \tilde{B}_{y}$ the undulator parameter, $\tilde{B}_{y}$ the on-axis magnetic flux density amplitude and $\gamma$ the electron's Lorentz factor. The same equation solved for $\gamma$ describes the resonance condition for a free electron laser (FEL),

$$
\gamma_{\mathrm{r}}=\sqrt{\frac{\lambda_{\mathrm{u}}}{2 \lambda}\left(1+\frac{K^{2}}{2}\right)},
$$

where $\gamma_{r}$ is the resonance energy of the FEL.

If the electron beam has a significant energy spread, the spectrum of the undulator radiation is broadened or, in case of a high gain FEL, the FEL gain is decreased. The energy spread of the beam can be considered significant if it approaches the bandwidth of the undulator radiation,

*axel.bernhard@kit.edu

Published by the American Physical Society under the terms of the Creative Commons Attribution 3.0 License. Further distribution of this work must maintain attribution to the author(s) and the published article's title, journal citation, and DOI. $\frac{\sigma_{\lambda}}{\lambda} \propto \frac{\lambda_{u}}{L_{\mathrm{u}}} \sim 10^{-2}$, or the bandwidth of the high-gain FEL amplifier, $\frac{\sigma_{\gamma}}{\gamma_{r}} \propto \frac{\lambda_{u}}{L_{\text {gain }}} \sqrt{\frac{L_{\text {gain }}}{z}} \sim 10^{-3} \sqrt{\frac{L_{\text {gain }}}{z}}$, respectively. Here $L_{\mathrm{u}}$ is the length of the undulator and $L_{\text {gain }}$ the gain length of the FEL.

In order to reduce the spectral broadening of the photon beam caused by the energy spread of the electron beam the following basic proposal of a transverse gradient undulator (TGU) was made [1]. Electrons with different energies enter the undulator at different transverse positions (e.g. $x$-positions). The poles of the undulator are tilted so that the magnetic flux density amplitude and in turn the undulator parameter also become a function of $x, K(x)=$ $\frac{e}{2 \pi m c} \lambda_{\mathrm{u}} \tilde{B}_{y}(x)$. If $\gamma(x)$ and $K(x)$ are matched to each other such that the modified undulator resonance equation

$$
\lambda=\frac{\lambda_{\mathrm{u}}}{2 \gamma^{2}(x)}\left(1+\frac{K^{2}(x)}{2}\right)=\mathrm{const}
$$

is satisfied for all particle energies, narrow bandwidth undulator radiation is emitted despite a large energy spread of the electron beam.

It was shown that a beam with zero emittance dispersed by a dogleg chicane consisting of two bending magnets followed by a realistic TGU can produce a photon beam with an energy bandwidth not exceeding the natural bandwidth of classical undulator radiation. That is technically possible even when the relative energy deviation of the electrons is $\frac{\Delta \gamma}{\gamma_{0}}= \pm 10 \%$ [2]. 
This relative energy range is used as target energy acceptance of the TGU throughout our model calculations presented below. It was estimated as a conservative upper limit covering both the typical single-shot energy spread and the shot-to-shot energy jitter of a laser wakefield accelerator (LWFA), based on beam measurements obtained with the LWFA at the JETI laser system in Jena, Germany [3].

The use of TGUs in a LWFA-driven FEL was recently proposed [4] and further investigated based on modified one-dimensional [5-8] and three-dimensional [9] FEL theory, using a simplified linear TGU model. In this simplified model linear approximations for the spectral dispersion of the electron beam $x=\eta \delta$ with $\eta$ the linear dispersion function and $\delta=\frac{\gamma-\gamma_{0}}{\gamma}$ and for the transverse gradient of the undulator parameter $K(x)=K_{0}(1+\alpha x)$ are applied. Under these assumptions the resonance condition Eq. (3) holds - in a further linear approximation-if

$$
\eta=\frac{2+K_{0}^{2}}{\alpha K_{0}^{2}}
$$

is satisfied, where $K_{0}=K\left[x\left(\gamma_{0}\right)\right]$.

For realistic scenarios these assumptions are impossible to fulfill in a strict way. The aim of this paper is therefore to investigate as a first step the spectral distribution of the incoherent undulator radiation for realistic and technically feasible TGU concepts. The achievable spectral bandwidth of the TGU radiation is analyzed in detail taking into account various general conditions of a realistic setup. In particular, these are the following six conditions: (i) the dispersion matching: the ideally required spectral dispersion of the electron beam depending on the TGU field gradient and the observation distance will be determined; (ii) the deflection of the particle trajectory inside the TGU due to the field gradient (so-called ponderomotive drift); (iii) the finite length of the undulator and its field termination; (iv) the finite observation distance; (v) observation through a finite aperture; and (vi) the finite transverse emittance of the incoming electron beam. We will show that and in which way these conditions determine the spectral distribution of the TGU radiation.

\section{POSSIBLE TGU LAYOUTS}

It was shown in [2] that transverse gradient undulators can be realized in various ways. The two technically most

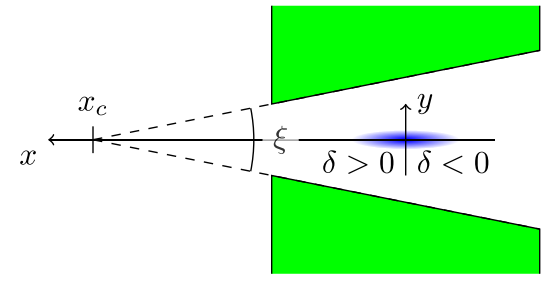

straightforward versions are sketched in Fig. 1: The transversely tapered and the cylindric TGU. The coordinate system used in this paper is also shown in Fig. 1 for both systems. Magnetic flux density calculations for both configurations were presented in [10].

The deflection strength gradient $\alpha_{K}=\frac{\partial K}{\partial x}=K_{0} \alpha$ is the key parameter for the TGU. To estimate the technically accessible range for this parameter we use the analytical expressions for the magnetic flux density derived in [10].

Restricting ourselves to the first term of the Fourier series expansion of the flux density in the deflection plane $(y=0)$, we get

$$
B_{y}(x, 0, z)=2 B_{\mathrm{pol}} \sin k_{\mathrm{u}} z \cos \xi / 2 e^{-k_{\mathrm{u}}\left(x_{c}-x\right) \sin \xi / 2}
$$

for the transversely tapered planar undulator and

$$
\begin{aligned}
B_{y}(x, 0, z)= & 2 \frac{B_{\text {pol }}}{K_{1}\left(k_{\mathrm{u}} r_{\text {cyl }}\right)} \sin k_{\mathrm{u}} z K_{1}\left(k_{\mathrm{u}} \rho\right) \cos \\
& \times\left(\arctan \frac{x_{c}-x}{r_{\text {cyl }}+h_{\text {gap }} / 2}\right)
\end{aligned}
$$

for the cylindric undulator. Here, $B_{y}(x, 0, z)$ is the flux density in the deflection plane, $B_{\text {pol }}$ the flux density at the undulator's pole surface, $k_{\mathrm{u}}=2 \pi / \lambda_{\mathrm{u}}, \xi$ the canting angle (full opening angle) of the transversely tapered undulator, $x_{c}$ the position of the imaginary crossing point of the tapered undulator halves or the position of the apex of the two cylinders, respectively. $K_{1}$ is the modified Bessel function of the second kind and

$$
\rho=\sqrt{\left(x_{c}-x\right)^{2}+\left(r_{\mathrm{cyl}}+\frac{h_{\mathrm{gap}}}{2}\right)^{2}}
$$

with $r_{\text {cyl }}$ the radius of the cylindric poles and $h_{\text {gap }}$ the gap height at the apex (see also Fig. 1). The resulting field amplitudes $\tilde{B}_{y}(x)=B_{y}\left(x, 0, \lambda_{\mathrm{u}} / 4\right)$ are shown in Fig. 2 . Note that $\tilde{B}_{y}(x)$ for the cylindric TGU exhibits an inflection point which can conveniently be chosen as $x=0$ and in the vicinity of which $\tilde{B}_{y}(x)$ is linear in good approximation whereas for the transversely tapered undulator $\tilde{B}_{y}(x)$ is curved for all $x$.

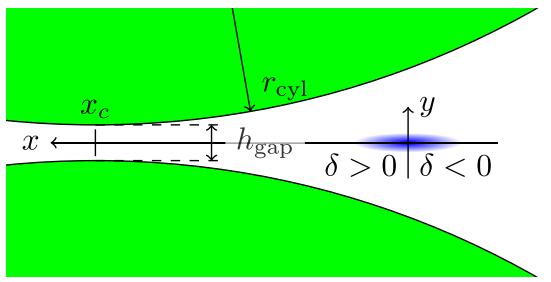

FIG. 1. Transverse gradient undulator geometries, coordinate systems and basic geometry parameters: left, transversely tapered TGU; right, cylindric TGU. 


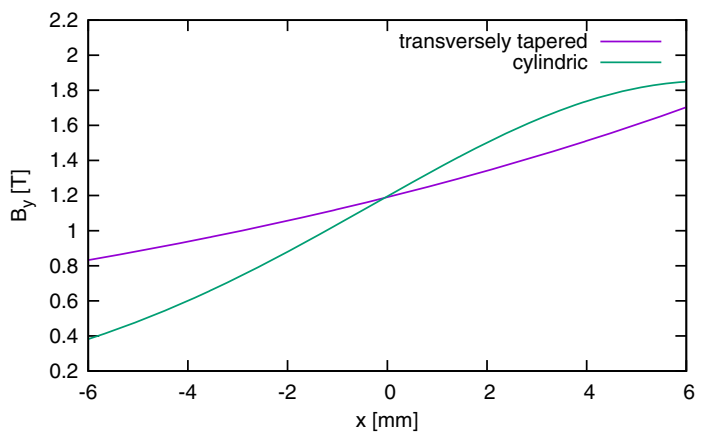

FIG. 2. Magnetic flux density amplitude as a function of transverse position $x$ for a transversely tapered and a cylindric TGU. Common parameters for both cases are $B_{\mathrm{pol}}=1.3 \mathrm{~T}$ and $\lambda_{\mathrm{u}}=10.5 \mathrm{~mm}$. For the transversely tapered undulator $x_{c}=13 \mathrm{~mm}$ and an opening angle $\xi=200 \mathrm{mrad}$ are assumed, for the cylindric undulator $r_{\text {cyl }}=30 \mathrm{~mm}$, $h_{\text {gap }}=1.1 \mathrm{~mm}$ and $x_{c}=6.47 \mathrm{~mm}$, corresponding to the example case discussed below.

From Eq. (5) the flux density gradient and in turn the deflection strength gradient in a transversely tapered undulator can directly be derived:

$$
\frac{\partial \tilde{B}_{y}}{\partial x}=\tilde{B}_{y} k_{\mathrm{u}} \sin \xi / 2 \quad \alpha_{K}=K_{0} k_{\mathrm{u}} \sin \xi / 2 .
$$

For the case of a cylindric undulator, it is more convenient to derive the gradient numerically from the calculated flux density amplitude. In [2] two cases at the edge of technical feasibility were discussed for this configuration.

If we consider short period undulators with $\lambda_{\mathrm{u}}<20 \mathrm{~mm}$, we see that the deflection strength gradient for realistic devices is limited to $\alpha_{K} \lesssim 100 \mathrm{~m}^{-1}$ for transversely tapered undulators, whereas gradients of 300 to $400 \mathrm{~m}^{-1}$ are achievable with superconducting cylindric undulators, depending on the superconductor technology applied. In the following we will use the example of a $\mathrm{Nb}$-Ti-based superconducting cylindric undulator.

\section{GENERAL CONSIDERATIONS AND METHODOLOGY}

\section{A. Limitations of the monochromaticity of TGU radiation}

In general there are two effects potentially limiting the monochromaticity of TGU radiation: a mismatch of the dispersion $x(\gamma, z)$ anywhere inside the TGU on the one hand, and the increased transverse electron beam size due to the spectral dispersion of the beam on the other hand.

The latter effect is not an issue if only the radiation in forward direction or in other words the radiation observed at infinite distance is considered, because if we think of the bunch as composed of zero-energy spread beamlets, the emittance of each individual of these beamlets is conserved and so is the monochromaticity of the radiation. At finite observation distances, however, the increased beam size translates to nonzero observation angles. Taking these into account, the resonance condition, Eq. (3), reads

$$
\lambda=\frac{\lambda_{\mathrm{u}}}{2 \gamma^{2}(x)}\left(1+\frac{K^{2}(x)}{2}+\gamma^{2}(x) \theta^{2}(x)\right),
$$

where $\theta$ is the observation angle which, if different from zero, causes a redshift of the observed radiation due to the relativistic Doppler effect. From Eq. (9) it is evident that the transverse gradient $K(x)$ can be employed to compensate also this effect. We will discuss below to which extent that is possible.

The perturbing effect of dispersion mismatch has several aspects. In our calculations we will determine the ideal position $x_{r}(\gamma)$ for the reference particles of each individual particle energy resulting in the same radiation wavelength for each particle energy and assume that the reference particles start at this transverse position either inside an infinitely long undulator or outside an undulator of finite length. Any horizontal displacement of particles from these reference trajectories will lead to a dispersion mismatch and thereby to a degradation of the monochromaticity of the TGU radiation which for any given $\gamma$ can be estimated in linear approximation from Eq. (3):

$\Delta \lambda=\left.\frac{\partial \lambda}{\partial x}\right|_{x_{r}} \Delta x=\left.\frac{\lambda_{\mathrm{u}}}{2 \gamma^{2}} K\left(x_{r}\right) \frac{\partial K}{\partial x}\right|_{x_{r}} \Delta x \quad$ with $\quad \Delta x=x-x_{r}$.

For a rough estimate of the severeness of the displacement of a particle from the ideal trajectory we may relate this approximated wavelength deviation to the natural bandwidth of the fundamental spectral line of the undulator radiation. A transverse particle displacement can in first order be viewed as tolerable if the condition

$$
\frac{K\left(x_{r}\right) \alpha_{K} \Delta x}{1+K^{2}\left(x_{r}\right) / 2} \ll \frac{1}{N_{\mathrm{u}}}
$$

is fulfilled.

Different types and sources of transverse displacements of particles from the reference orbit may be distinguished and will be analyzed in this paper.

First, the electron bunch as a whole may be deflected and/or displaced inside the TGU. To avoid a deflection at the entrance of a TGU of finite length, a well-adapted field termination is of crucial importance.

There are two further potential sources of a deflection of the whole beam inside even an infinitely long TGU, the ponderomotive force and local field errors caused by mechanical imperfections. 
The ponderomotive force necessarily results from the oscillatory motion of the particles in a field with transverse amplitude gradient. The drift driven by this force can be considerably large and has to be corrected by the superposition of a correction field. In realistic TGUs, particularly TGUs of finite length, this correction cannot be realized in an ideal way. Residual displacements from the ideal trajectory due to the ponderomotive force will always, though minimized, be present.

The effect of spurious deflections inside an undulator due to local field errors is normally evaluated in terms of the rms-phase error [11]. This concept, developed for planar undulators, in principle still holds for transverse gradient undulators. However, the usual calculation method based on field integrals is not sufficient in this case. Because of the dependence of the magnetic flux density on the transverse position of the particle it is necessary to fully track the particle through the TGU. We will omit a detailed discussion of local field errors in this paper.

Finally, apart from possible displacements of the electron bunch as a whole, also the transverse displacements of the individual particles from the reference trajectories affect the monochromaticity of the TGU radiation. This effect necessarily occurs when the electron bunch has a finite emittance and depends on the choice of the optical functions at the entrance of the TGU. Similar to the treatment of the dispersion we will consider the bunch as composed of monoenergetic beamlets of equal finite emittance and individually adjustable optical functions.

To achieve a good matching between the beam properties and the TGU in reality, it will in general be necessary to conjointly optimize the optics of the beam transport line, the field termination of the undulator and the trajectory correction inside the undulator with the aim of minimizing the average displacement of the individual particles from their ideal trajectory. The layout of the transport line optics is a very demanding task as discussed in [12]. We will not treat this topic here but rather focus on a quantitative analysis of the perturbing effects described above and in this way on deriving reliable conditions for the transport line matching.

\section{B. General remarks on the investigation method}

For our model calculations we will use the example case of a short-period cylindric TGU which has been developed and built at KIT for a proof-of-principle experiment at the LWFA at the JETI high-power laser in Jena, Germany $[13,14]$. Our calculations were performed with WAVE [15]. This code calculates the radiation field from the particle trajectories determined by symplectic particle tracking through the TGU field. The field maps of the undulator were calculated with the finite-element-code OPERA3D [16]. The electron beam is represented by monochromatic beamlets or macroparticles at discrete energies. The bandwidth and maximum intensity of the resulting radiation is used as the figure of merit for evaluating the TGU concept.

\section{TGU and electron beam model}

The example case discussed in the following is an idealized model of the aforementioned superconducting TGU (SCTGU) realized at KIT. The model is idealized in the sense that we disregard certain implications of our particular technical design like e.g. peculiarities of our winding scheme for the superconducting coils.

The example undulator is an iron-free superconducting undulator with cylindric pole shape shown in Fig. 3. The geometrical and magnetic parameters are summarized in Table I. For the investigation of the effects of the ponderomotive drift, the finite observation distance and the finite electron beam emittance we consider this undulator to be 100 periods out of an infinitely long device, i.e. we disregard the field termination and start our particles inside the undulator. The parameters in Table I are optimized for the (nonlinear) dispersion of a simple two-dipole chicane, assuming a central beam energy of $E_{0}=120 \mathrm{MeV}$ and a bandwidth of the beam energy of $\frac{\Delta E}{E_{0}} \pm 10 \%$.

Assuming such a large bandwidth, the beam has to be spectrally dispersed over roughly $\Delta x= \pm 2 \mathrm{~mm}$. As discussed in $[2,13]$, the correction field required for the suppression of the ponderomotive drift is in good approximation parabolic in $x$. In reality this correction field will be generated by two long racetrack coils placed inside the undulator coil former. This former was chosen to be nonmagnetic in order not to perturb the correction field. Figure 4 shows the geometry of the corrector magnet and the on-axis correction field as a function of $x$. Uncorrected and corrected relative trajectories for $E=108,120$ and $132 \mathrm{MeV}$ will be shown below.

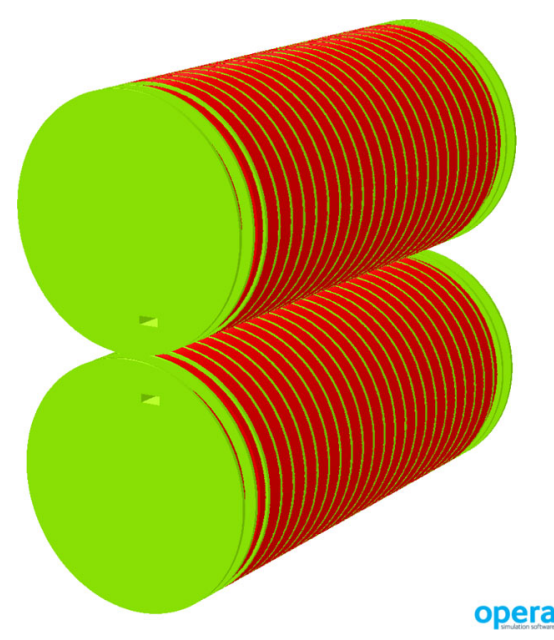

FIG. 3. Model of the cylindric superconducting TGU, shortened to ten periods for better visibility. The superconducting coils are depicted in red, the copper coil former in green. 
TABLE I. Parameters of the example TGU.

\begin{tabular}{lccc}
\hline \hline Period length & $\lambda_{\mathrm{u}}$ & {$[\mathrm{mm}]$} & 10.5 \\
Number of full periods & $N_{\mathrm{u}}$ & & 100 \\
Pole radius & $r_{\mathrm{cyl}}$ & {$[\mathrm{mm}]$} & 30 \\
Gap width on symmetry axis & $g$ & {$[\mathrm{~mm}]$} & 1.1 \\
Shift symmetry axis-beam center & $x_{c}$ & {$[\mathrm{~mm}]$} & 6.47 \\
Gap width at beam center & $g_{c}$ & {$[\mathrm{~mm}]$} & 2.4 \\
Flux density amplitude at beam center & $\tilde{B}_{y 0}$ & {$[\mathrm{~T}]$} & 1.10 \\
Undulator parameter at beam center & $K_{0}$ & & 1.07 \\
Transverse gradient & $\alpha_{K}$ & {$\left[\mathrm{~m}^{-} 1\right]$} & 149.5 \\
Relative transverse gradient & $\alpha$ & {$\left[\mathrm{m}^{-} 1\right]$} & 139.7 \\
\hline \hline
\end{tabular}

On the side of the electron beam, the following idealized assumptions are made: the beam consists of 21 parallel, monochromatic and-in the first step-zero-emittance beamlets at equidistant discrete energy levels in the interval 108 to $132 \mathrm{MeV}$. The photon spectra for each case discussed in this paper are calculated for a beam current of $10 \mathrm{pA}$, referring to a bunch charge of $10 \mathrm{pC}$ at a repetition rate of $1 \mathrm{~Hz}$ typically achieved in state-of-the-art laser wakefield accelerators. For the calculation of the photon spectra emitted by individual monoenergetic beamlets a bunch current of $10 / 21 \mathrm{pA}$ is assumed for each beamlet, in order to facilitate the comparison with incoherently added spectra.

\section{MODEL CALCULATION RESULTS}

\section{A. Basic proof of concept, dispersion matching}

Figure 5 shows radiation spectra observed at $1 \times 10^{5} \mathrm{~m}$ distance (in the following referred to as infinite distance) and the corresponding dispersion functions $\left(\eta^{\prime}=0\right.$ is always assumed) for a planar undulator, an ideally linear
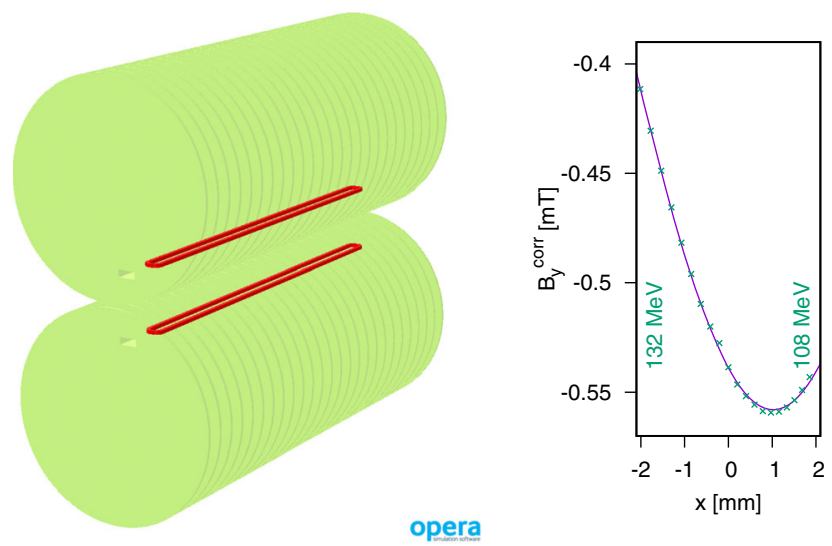

FIG. 4. Geometry of the correction coils generating an $x$ dependent magnetic field correcting for the ponderomotive drift in the example-SCTGU (left). The correction field generated by this coil configuration in the deflection plane is shown in the right part of the figure (solid line). The single data points shown in the graph indicate the required correction field calculated from the displacement of the electrons after one period, if uncorrected.
TGU and the cylindric SCTGU for equal deflection parameters $K_{0}$ and, where applicable, deflection parameter gradients, applying linear and nonlinear dispersion matching conditions. The spectra shown are the on-axis spectral photon flux densities for each beamlet energy as a function of relative deviation from the radiation wavelength $\lambda\left(E_{0}\right)$, which is in our example case $\lambda(120 \mathrm{MeV})=147.6 \mathrm{~nm}$. To facilitate the comparison of the calculation results for different observation distances, photon flux densities are expressed in units of $1 /\left(\mathrm{s} \mathrm{mrad}^{2} 0.1 \%\right.$ bandwidth $)$.

This calculation in principle proves the validity of the concept, both for the ideal linear TGU and for the cylindric SCTGU. The spectral full-width-half-maximum is reduced from $40 \%$ in the case of the planar undulator to $0.9 \%$ in the case of the SCTGU. However, the calculation also shows that the above considerations based on Eq. (3) and the
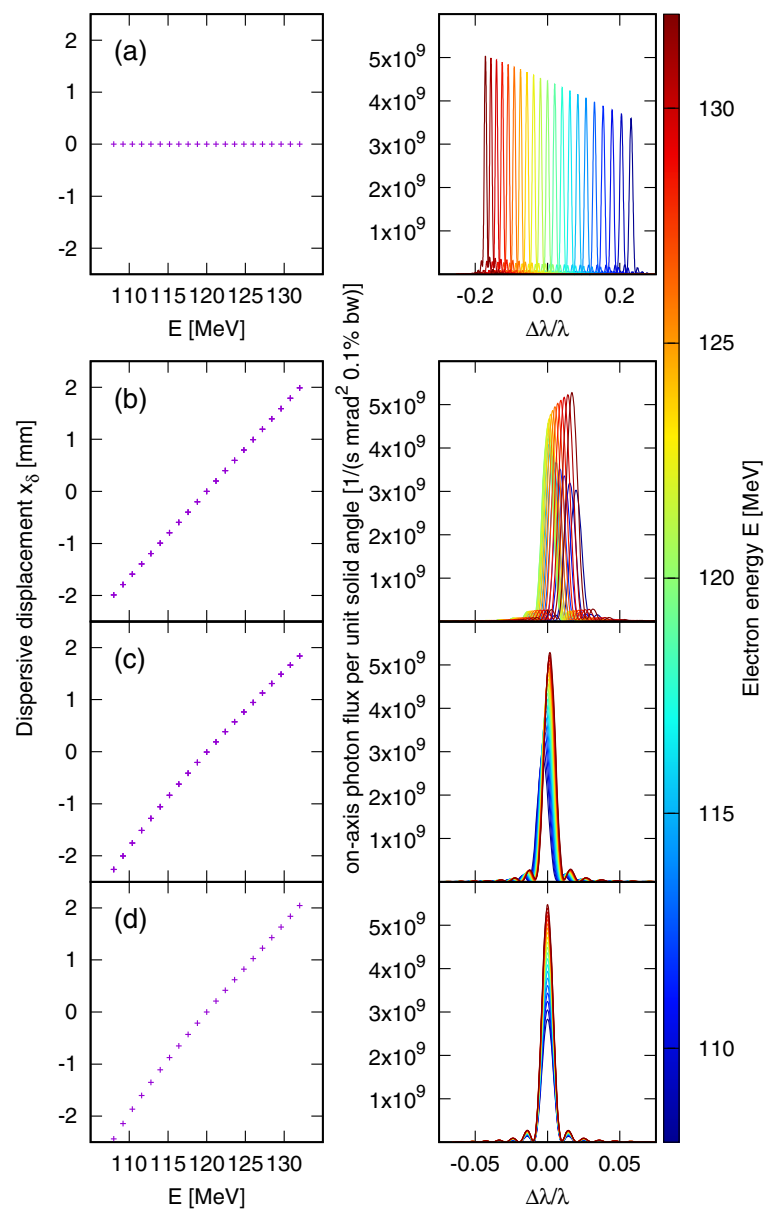

FIG. 5. Basic proof of the TGU concept: Electron energy dispersion and resulting radiation spectra for an electron energy band of $\Delta E=120 \mathrm{MeV} \pm 10 \%$ for the cases (a) planar undulator with $K=1.07$, (b) ideal linear TGU with $K_{0}=1.07$ and $\alpha=$ $139.7 \mathrm{~m}^{-1}$ and linear dispersion [Eq. (4)], (c) the same with nonlinear dispersion according to Eq. (12), (d) idealized cylindric SCTGU with optimized dispersion. For all cases a beam current of $10 \mathrm{pA}$, equally distributed over the 21 monoenergetic zeroemittance beamlets, is assumed. 
linear approximation for the resonance condition, Eq. (4), are sufficient only for a very limited energy band around $E_{0}$. Solving Eq. (3) and $K(x)=K_{0}(1+\alpha x)$ for $x$ exactly, yields

$$
x_{r}(\gamma)=-\frac{1}{\alpha}\left(1-\sqrt{1+\frac{\left(\gamma / \gamma_{0}-1\right)\left(2+K_{0}^{2}\right)}{K_{0}^{2}}}\right) .
$$

Figure 5(c) shows the spectra resulting for the linear TGU if this exact dispersion relation is applied. The dispersion and spectra shown in Fig. 5(d) for the SCTGU result from an optimization of the electron beamlet starting positions using the monochromaticity of the radiation as the figure of merit. Note again that neither of the dispersions shown in the figure result from a particular beam transport optics but rather define the dispersion matching condition for that optics.

\section{B. Ponderomotive drift}

As the first potentially disturbing effect occurring already in ideal TGUs we investigate the ponderomotive particle drift.

This drift is caused by the difference of the deflecting field the particle experiences along its oscillatory path depending on whether it is moving to positive or negative $x-x_{r}$. For an estimate for this effect we consider the Lorentz force equation for the reference particle with the linear approximation for the transverse field gradient

$$
\ddot{x}=-\frac{e}{\gamma m} \tilde{B}\left(x_{r}\right)(1+\alpha x(z)) \cos \left(k_{\mathrm{u}} z\right) \dot{z},
$$

which can be integrated and translated to the equation for the particle's deflection angle assuming the weak undulator condition $\dot{z}=\beta c$ :

$$
x^{\prime}=\frac{\dot{x}}{\dot{z}}=-\frac{e \tilde{B}\left(x_{r}\right)}{\gamma m \beta c} \int[1+\alpha x(z)] \cos k_{\mathrm{u}} z \mathrm{~d} z
$$

Evaluating the integral for one undulator period and approximating $x(z)$ under the integral by the trajectory in a plane weak undulator $x(z)=\frac{K\left(x_{r}\right)}{\beta \gamma k_{\mathrm{u}}} \cos k_{\mathrm{u}} z$ yields a firstorder estimation of the ponderomotive deflection after one undulator period,

$$
x_{1}^{\prime}=-\frac{\pi K\left(x_{r}\right)}{\gamma^{2} \beta^{2} k_{\mathrm{u}}} \alpha_{K}
$$

For a conservative estimation of the ponderomotive drift after $N_{\mathrm{u}}$ periods we may assume that the particles are deflected by the same amount in each period. The net displacement of the beam is then given by
$\Delta x_{N_{\mathrm{u}}}=\sum_{i=2}^{N_{\mathrm{u}}}(i-1) x_{1}^{\prime} \lambda_{\mathrm{u}}=x_{1}^{\prime} \lambda_{\mathrm{u}} \frac{\left(N_{\mathrm{u}}-1\right)\left(N_{\mathrm{u}}-2\right)}{2}$.

Obviously this "natural" ponderomotive displacement grows very quickly with the number of undulator periods and we expect a severe impact on the monochromaticity of the TGU radiation. Therefore schemes for the correction of the ponderomotive drift by superposition of a correction field depending on the transverse coordinates and constant in $z$ were introduced.

To prove the validity of the correction schemes for the ideal linear TGU introduced in [9] as well as for our example case described above, we compare the radiation spectra for both cases with and without correction of the ponderomotive drift.

Figure 6 shows the particle trajectories through a 100period TGU for three particle energies (in the graphs the respective starting positions in $x$ were shifted to zero) in a linear TGU and in the cylindric SCTGU with and without the correction field applied. The transverse particle displacement over 100 periods is considerably large: $>600 \mu \mathrm{m}$ as compared to the $\Delta x \ll 134 \mu \mathrm{m}$ suggested as tolerable by Eq. (11).

Quite surprisingly, however, the spectral bandwidth of the radiation emitted in the forward direction is virtually
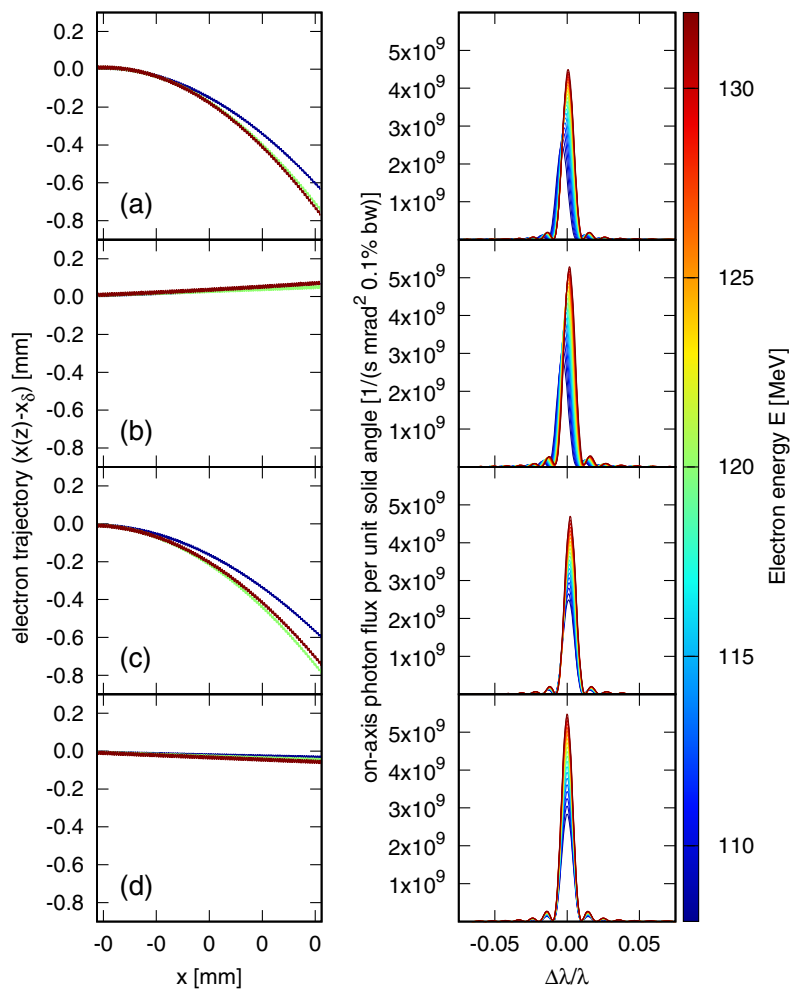

FIG. 6. The effect of the ponderomotive particle drift in TGUs: (a) linear TGU without trajectory correction and (b) with correction, (c) cylindric SCTGU without trajectory correction and (d) with correction. 
unaffected by this displacement. A detailed analysis of the phase relation between electron and photon along the calculated electron trajectories shows that the photon's phase slip between longitudinal positions of the electron with transverse velocity component $v_{x}=0$ remains constant exactly like in a planar undulator, in spite of the transverse particle drift.

At observation points at finite distance, this picture changes due to the relativistic Doppler shift, which makes a correction of the ponderomotive drift still favorable. For applications requiring long (FEL) or transparent (storage ring) TGUs, a correction of the transverse drift is of course indispensable.

\section{Finite undulator length}

As in the case of planar undulators, the magnetic field of a real finite transverse gradient undulator is required to be terminated in such a way that the reference particle-in our case the respective dispersion reference particles for each electron energy — enters and exits the undulator under zero angle and zero transverse displacement. In planar undulators this condition is equivalent with the condition that the first and second field integral along the beam axis vanish, which is achieved by terminating the undulator with a matching period with $1 / 4$ and $3 / 4$ of the field amplitude at the first/last and second/next to last pole, respectively.

In superconducting undulators with vertical coils, the matching condition can in good approximation be fulfilled by reducing the number of Ampère turns in the first/last and second/next to last winding package to $1 / 4$ and $3 / 4$ of that in the main periods, respectively. This matching scheme turns out to work also for the ideal cylindric SCTGU with the trajectory correction described above, requiring, however, a readjustment of the trajectory correction field which can technically be achieved easily by adjusting the current in the correction coils.

Figure 7 shows the required spectral dispersion and the spectra of the emitted radiation for a 100-period SCTGU employing this matching scheme, compared to the so far considered 100-period section of an infinitely long SCTGU. The spectral quality of the emitted radiation can fully be recovered even without a significant modification of the dispersion matching.

\section{Observation at finite distance}

So far we have dealt with radiation spectra observed at a point at virtually infinite distance on the $z$-axis or, in other words, under virtually zero observation angle for each electron energy. That means that our conclusions on the spectral photon flux density drawn so far hold also for any photon beam optics selecting the parallel radiation with a small angular acceptance (as for example Bragg optics or gratings do) placed at a finite distance from the source.

If, in contrast, the observation at finite distance with an energy-dispersive detector with a finite aperture and a
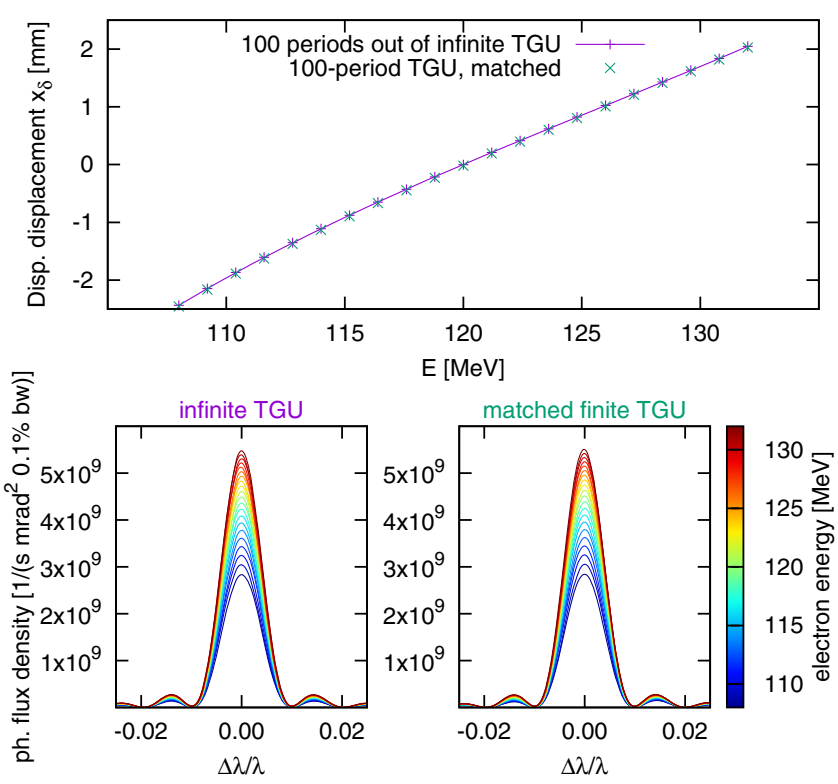

FIG. 7. Comparison of the required dispersion and the resulting radiation spectra (assuming zero-emittance beamlets) between the case of 100 periods out of an infinitely long SCTGU and the case of a finite 100-period SCTGU with matching periods. See the text for details.

virtually unlimited angular acceptance (like for example Silicon Drift Detectors or $\mathrm{Si}(\mathrm{Li})$ detectors used in x-ray spectroscopy) is considered, the picture changes. In that case the finite spectral dispersion of the electron beam results in finite observation angles and in turn to a redshift of the radiation spectrum emitted by the parts of the beam with $E \neq E_{0}$ due to the relativistic Doppler effect as discussed above and described by Eq. (9). This effect is-obviously-the more severe, the closer the observation point to the source and the larger the spectral dispersion of the beam, i.e. the broader the spectrum of the beam and/or the smaller the transverse field gradient of the TGU. As we talk about "laboratory-scale" radiation sources, applying the generated radiation at relatively short distances behind the source is definitely part of the concept.

The transverse field gradient can, however, be employed to compensate not only the energy spread of the electron beam, but also the relativistic Doppler shift by an appropriate adjustment of the electron beam dispersion. Figure 8 shows an extreme example using the limit case of an energy-dispersive point detector. The bottom right graph shows the radiation spectra calculated for our example TGU and an observation point at $2 \mathrm{~m}$ distance from the center of the undulator with reoptimized starting positions of the monoenergetic electron beamlets. The top graph shows the corresponding dispersion in comparison to that for the observation at infinite distance. Significantly larger higher-order dispersion terms are required in this case.

The spectral width of the radiation is basically recovered: The relative full-width-half-maximum of the incoherently 


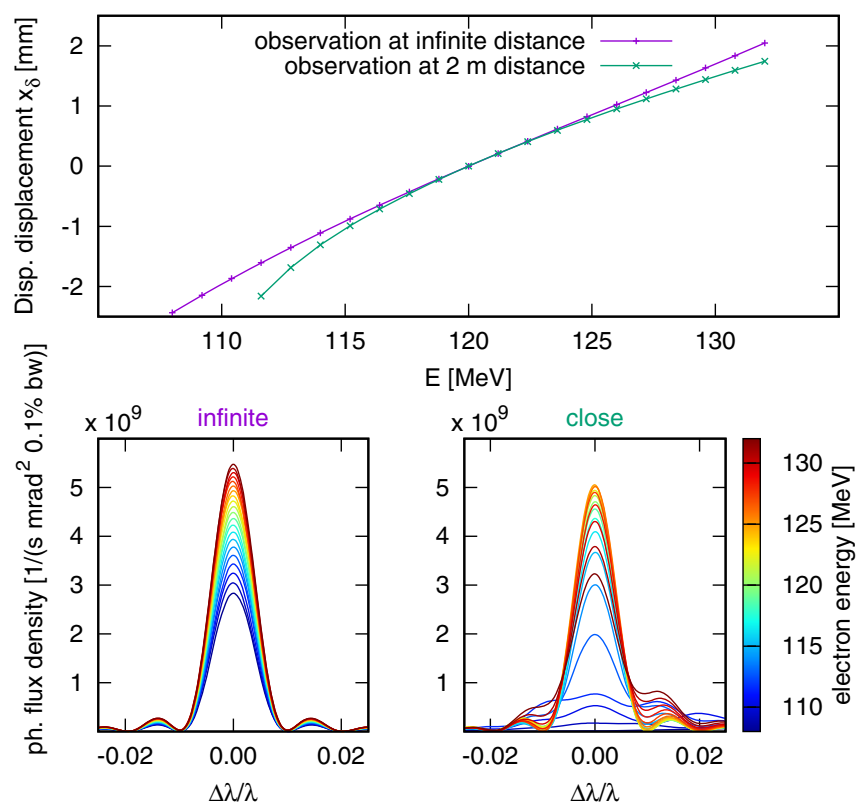

FIG. 8. Comparison of the optimized spectral dispersion of the electron beam and resulting radiation spectra for an observation point in infinite and $2 \mathrm{~m}$ distance, respectively.

summed-up spectrum is $0.90 \%$ as compared to $0.88 \%$ for the spectrum observed at infinite distance. The peak flux density (expressed in photons per unit solid angle and $0.1 \%$ relative bandwidth) is slightly decreased: $8 \times 10^{10} \mathrm{mrad}^{-2}$ as compared to $9 \times 10^{10} \mathrm{mrad}^{-2}$. The intensity decrease is due to the fact that the compensation of the Doppler shift does not work equally well for all electron energies. In particular, the electrons at the low-energy end of the spectrum hardly do contribute to the observed radiation any more. That can be understood looking at the required spectral dispersion of the electrons: on the low-energy side, the dispersive displacement needs to be increased in order to compensate the Doppler shift, which in turn is further increased, too. A fundamental limit of this compensation scheme is reached once the Doppler shift grows faster than the wavelength shift due to the transverse gradient of the undulator field, which in our example is the case for an electron energy of about $112 \mathrm{MeV}$.

As mentioned, for a given transverse field gradient in the TGU and a given spectral width of the electron beam the Doppler effect is the less pronounced the larger the distance to the observation point is. The graphs on the left-hand side of Fig. 9 show that in our example case the necessary adjustment of the electron beam dispersion as well as the peak intensity drop become significant at observation distances lower than $5 \mathrm{~m}$. For comparison, the plotted peak intensities are expressed as spectral photon flux per solid angle and normalized to the peak value of the flux density spectrum for $x_{\mathrm{obs}}=0, E=132 \mathrm{MeV}$ and $z_{\mathrm{obs}}=1 \times 10^{5} \mathrm{~m}$.

Since the capability of compensating the Doppler shift is limited particularly in the low-energy part of the electron

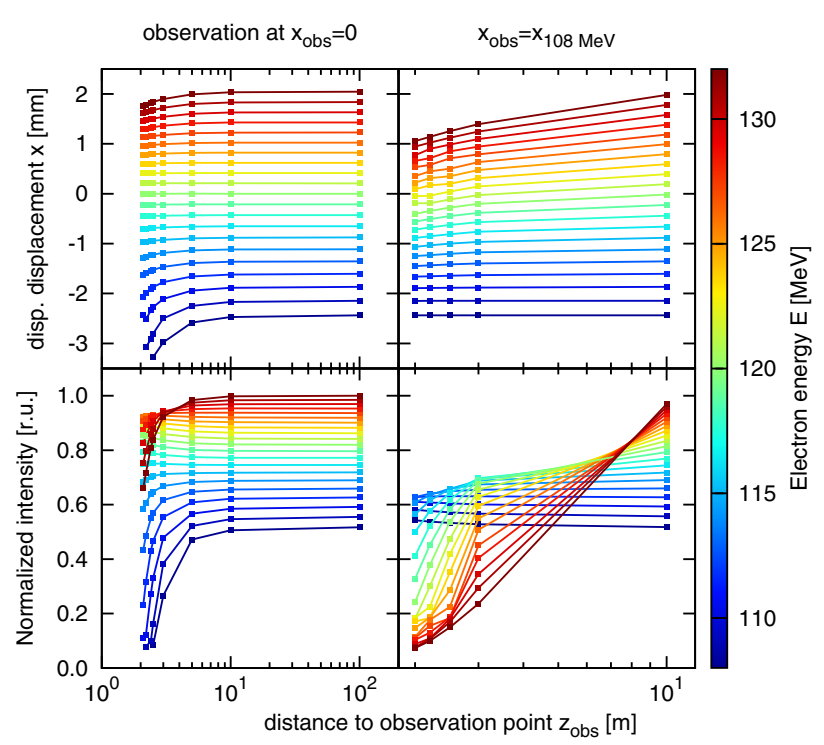

FIG. 9. Optimized electron dispersion and normalized peak intensity of the resulting radiation for each electron energy as a function of distance to the observation point for two different transverse positions of the observation point. For details on the intensity normalization refer to the text.

spectrum, it is tempting to additionally transversely shift the observation point to some $x<0$ in order to reduce the Doppler shift for the low-energy electrons. On the righthand side of Fig. 9 the dispersion and radiation intensity as a function of distance are shown for an observation point opposite to the transverse position of the $108 \mathrm{MeV}$ beamlet which represents the low-energy end of the electron spectrum. In this case it is indeed possible to compensate the Doppler shift for all beamlets at all observation point distances considered, however at the cost of a strongly decreasing radiation intensity in the medium- and highenergy part of the electron spectrum.

\section{E. Photon flux through finite aperture}

The cases considered in the previous section are limit cases in the sense that they assume an infinitely small angular or spatial acceptance, respectively, of the system detecting the spectral distribution of the TGU radiation. To describe more realistic conditions, the key characteristics of undulator radiation are typically expressed in terms of photon flux through a finite aperture (or into a finite solid angle), or in terms of spectral brightness, the spectral photon flux integrated over the full forward solid angle and divided by the source size and divergence.

Figure 10 shows an example for the photon flux emitted by the SCTGU and collected through a finite aperture at infinite and $2 \mathrm{~m}$ distance, respectively, in comparison to the planar undulator. For the SCTGU the spectral dispersion of the electron beam shown in Fig. 8 is assumed. The aperture for all cases was chosen to correspond to a solid angle of $1.7 \mathrm{mrad} \times 1.7 \mathrm{mrad}$ or $\left(\frac{0.4}{\gamma}\right)^{2}$ for $120 \mathrm{MeV}$, similar to the 

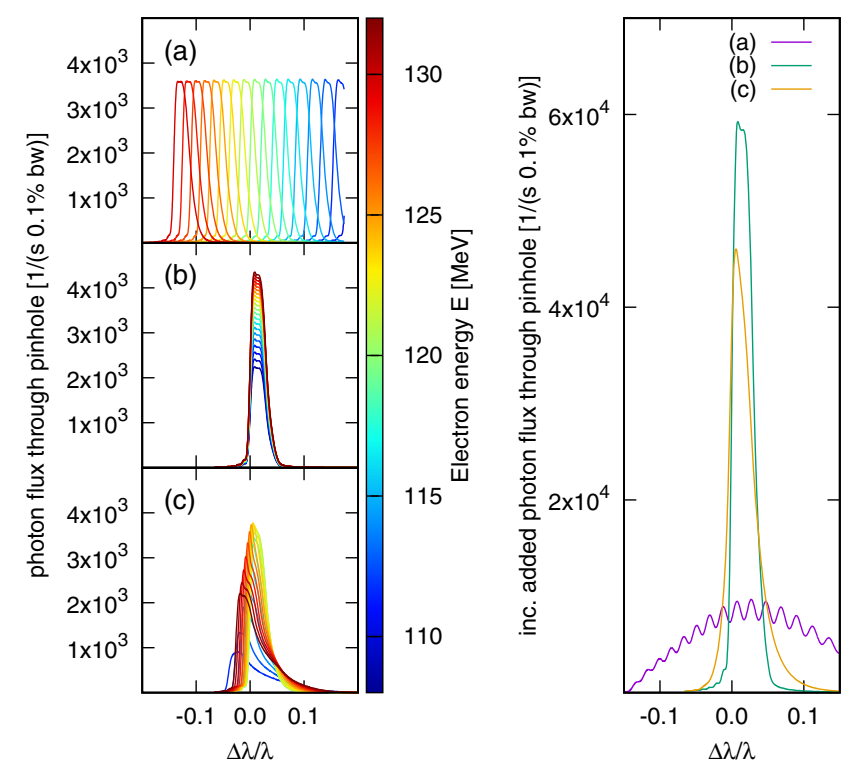

FIG. 10. Left: Spectra of the photon flux through a pinhole with $1.7 \mathrm{mrad} \times 1.7 \mathrm{mrad}$ angular acceptance for (a) a planar undulator, (b) the example SCTGU observed at infinite distance, (c) the SCTGU at $2 \mathrm{~m}$ distance; right: incoherently added spectra for the three cases assuming a Gaussian energy distribution of the electrons with $\sigma_{E}=6 \mathrm{MeV}$.

choice e.g. of a $1 \mathrm{~mm} \times 1 \mathrm{~mm}$ pinhole at $30 \mathrm{~m}$ distance for the European Synchrotron Radiation Facility.

The integration over a finite solid angle leads to a broadening of the spectrum for each electron energy. For the case of the planar undulator as well as for that of the TGU with an observation point at infinite distance, the onaxis flux density spectra shown in the previous section define the short-wavelength edge of the broadened spectra. As these edges are perfectly matched in this case, the result obtained for the flux density turns out to be equally valid also for the flux through a finite aperture.

Regarding the TGU photon flux spectra observed at $2 \mathrm{~m}$ distance, the additional influence of the finite observation angle for the off-energy beamlets is visible, which shifts the short-wavelength edges and maxima of the spectra to slightly shorter wavelengths. The integration over a finite aperture therefore relaxes the condition for the redshift compensation discussed in the previous section.

The incoherently added spectra shown in the right panel of Fig. 10 show that for the given example the spectral photon flux through a finite aperture emitted by the TGU is significantly increased as compared to that emitted by a planar undulator. The achievable gain depends on both, the observation distance and the size of the aperture.

We note that on the other hand at the same time the source size is increased through the spectral dispersion of the electron beam. We can estimate from Eqs. (4) and (11) that the source size must increase at least by a factor of $2 N_{\mathrm{u}} \frac{\sigma_{E}}{E}$, where $\sigma_{E} / E$ is the relative energy spread of the electron beam. If we further estimate the maximal possible gain in flux through the ratio between the spectral width of planar undulator radiation for the given energy spread of the electron beam on the one hand and the ideal spectral width of the undulator radiation on the other hand,

$$
\frac{\sigma_{\lambda, \text { planar }}\left(\sigma_{E}\right) / \lambda}{1 / N_{\mathrm{u}}}=2 N_{\mathrm{u}} \frac{\sigma_{E}}{E},
$$

we see that in general the spectral brightness of the generated radiation is not affected by the TGU, while the photon flux through a finite aperture is increased.

\section{F. Finite beam emittance}

As discussed above, a finite electron beam emittance necessarily leads to a spectral broadening of the TGU radiation and in turn to a decrease in peak intensity. Referring to the resonance condition, Eq. (11), we expect, however, this effect to be minor if the beamlet envelope fulfills the condition

$$
\sqrt{\beta_{x}(\gamma) \epsilon_{x}(\gamma)} \ll \frac{1+\frac{K^{2}}{2}}{N_{\mathrm{u}} K \alpha_{K}}
$$

everywhere in the TGU, with $\beta_{x}$ the betatron function and $\epsilon_{x}$ the geometric emittance. Additionally, a finite beam emittance implies a finite angular divergence of the electron beam, leading to a redshift of the radiation emitted by particles with a finite transverse momentum due to the nonzero observation angle as described by Eq. (9). From that a general constraint on the electron beam divergence inside the undulator may be derived:

$$
\frac{\epsilon_{x}(\gamma)}{\beta_{x}(\gamma)} \ll \frac{1+\frac{K^{2}}{2}}{N_{\mathrm{u}} \gamma^{2}} .
$$

For our example case at the central beam energy $E_{0}=$ $120 \mathrm{MeV}$ these conditions read

$$
\sqrt{\beta_{x}(\gamma) \epsilon_{x}(\gamma)} \ll 10^{-4} \mathrm{~m}, \quad \sqrt{\frac{\epsilon_{x}(\gamma)}{\beta_{x}(\gamma)}} \ll 0.35 \mathrm{mrad} .
$$

This estimation is basically confirmed by the WAVE calculations performed again for 100 periods out of the infinitely long idealized cylindric TGU.

Figure 11 shows results of calculations for a monoenergetic beamlet $(E=120 \mathrm{MeV})$ consisting of 500 macroparticles. The left part of the figure shows the phase space distributions at three longitudinal positions (entrance, center and exit of the undulator) for a beam with an isotropic geometric emittance of $\epsilon_{x}=\epsilon_{y}=10^{-8} \mathrm{~m} \mathrm{rad}$. The vertical beta function $\beta_{y}$ is chosen such that it takes a constant value along the undulator due to the undulator's focusing properties in the vertical direction. In the deflection direction, a beta function $\beta_{x}$ symmetric to the center of 

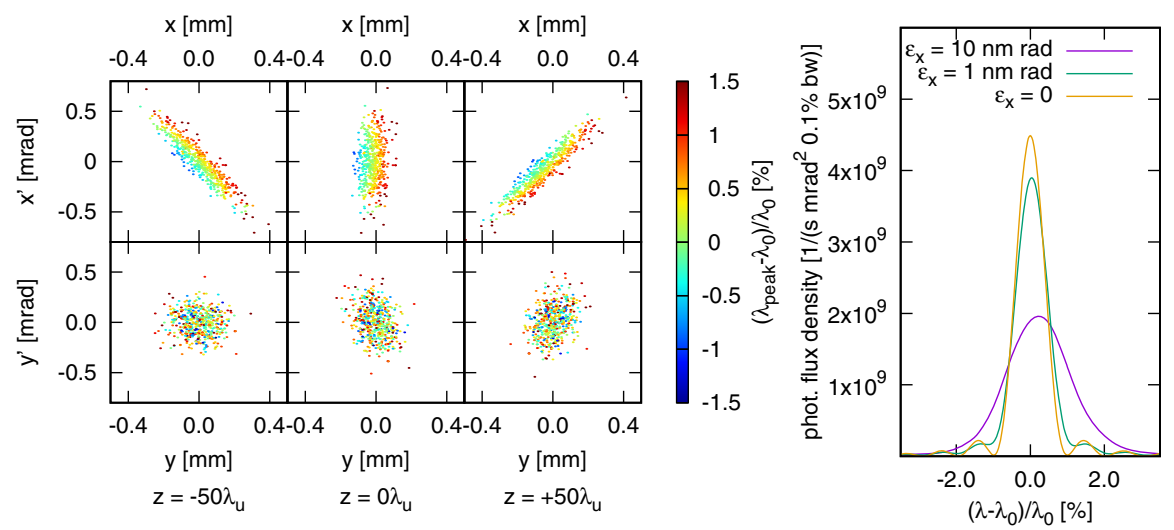

FIG. 11. Left: Phase space distribution in $x$ and $y$ of a $120 \mathrm{MeV}$ electron bunch at the entrance, center and exit of the idealized cylindric TGU. The electron bunch parameters were chosen as $\beta_{x}^{\text {waist }}=0.5 \mathrm{~m}, \quad \beta_{y}=0.7 \mathrm{~m}=$ const and the geometric emittance $\epsilon_{x}=\epsilon_{y}=10^{-8} \mathrm{mrad}$. The color scale refers to the relative deviation of the peak energy of the radiation emitted by the respective particle with respect to that of the reference particle. Right: Incoherently added radiation spectrum emitted by the particle bunch as a whole for different values of $\epsilon_{x, y}$, observed at infinite distance.

the undulator is chosen which at the beam waist takes a value of $\beta_{x}^{\text {waist }}=L_{\mathrm{u}} / 2=0.5 \mathrm{~m}$. The full sigma width of the beam envelope at the waist in this case is $1.4 \times 10^{-4} \mathrm{~m}$, slightly greater than the limit stated in Eq. (18). Accordingly, the incoherently added radiation spectrum emitted by this particle bunch shown in the right-hand part of Fig. 11 exhibits a spectral width (FWHM) which is by a factor of 2 greater than that of the zero-emittance beam shown for comparison. In addition, the spectrum is slightly shifted towards longer wavelengths. Reducing the geometric emittance by an order of magnitude leads, as expected, to a radiation spectrum with nearly the natural bandwidth, as also shown in the figure.

For a better understanding of the role of the beta function the macroparticles shown in the left part of Fig. 11 are color coded with respect to the relative deviation of the wavelength of their individual radiation spectrum from that of the zero-emittance spectrum. It can be observed that the particle's radiation spectrum is basically uncorrelated with its coordinates in the $y y^{\prime}$ phase space, while strongly correlated to that in the $x x^{\prime}$ phase space, as expected. Putting the focus on the particle distribution at the beam waist first, also this picture looks very much as expected: The wavelength of the radiation emitted by the particles is mainly correlated to their $x$-coordinate and only weakly depends on their transverse momentum $x^{\prime}$. On the path through the undulator, this "striped" color pattern is rotated together with the phase space ellipse. That in turn means that in first order approximation the spectral broadening of the TGU radiation due to the finite beamlet size is mainly determined by the width of the beam envelope at the beam waist, i.e. small values of $\beta_{x}^{\text {waist }}$ are favored.

This conclusion is confirmed by Fig. 12, where the spectrum for $\epsilon_{x}=10^{-8} \mathrm{~m} \mathrm{rad}$ shown in Fig. 11 is compared to that calculated for $\beta_{x}^{\text {waist }}=0.2 \mathrm{~m}$ with the same beam parameters in all other respects. Of course, at some point, the weaker dependence of the radiation spectrum on the $x^{\prime}$-coordinate starts to play a role. In our example case the choice of $\beta_{x}^{\text {waist }}=0.2 \mathrm{~m}$ turns out to be optimal [12].

Another way of looking at the impact of a finite beam emittance on the radiation of a TGU particularly useful for the design of the beam transport system is to inspect the dynamical acceptance. The dynamical acceptance can be defined as the area in the transverse phase space at the entrance of the undulator for which the wavelength of the emitted radiation is within a certain defined bandwidth around that emitted by the reference particle. This area may be further confined by demanding a certain peak intensity level. Figure 13 shows acceptance levels to this effect for our example case (100 periods out of the infinitely long TGU) and an electron energy of $120 \mathrm{MeV}$. Since the above discussion suggests that the dynamical $y y^{\prime}$-acceptance

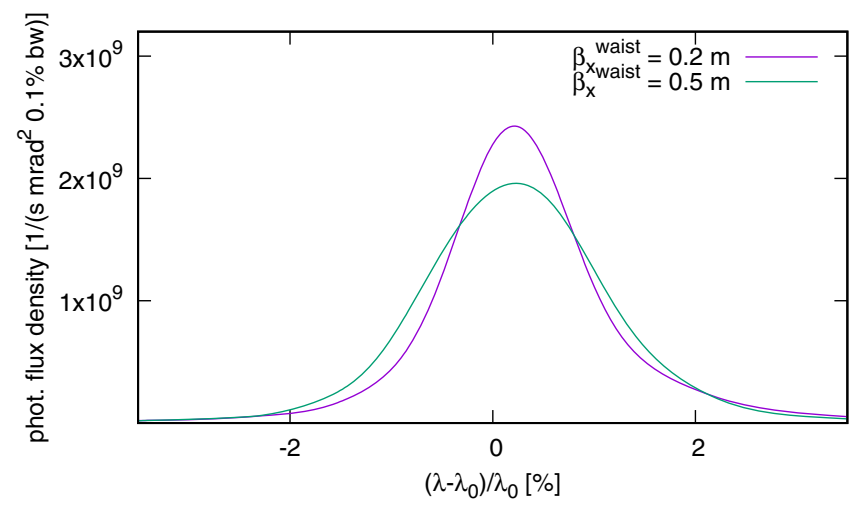

FIG. 12. Incoherently added radiation spectra emitted by particle bunches with $E=120 \mathrm{MeV}, \epsilon_{x}=10^{-8} \mathrm{~m} \mathrm{rad}$ for two different choices of $\beta_{x}^{\text {waist }}$, observed at $10^{5} \mathrm{~m}$ distance. 

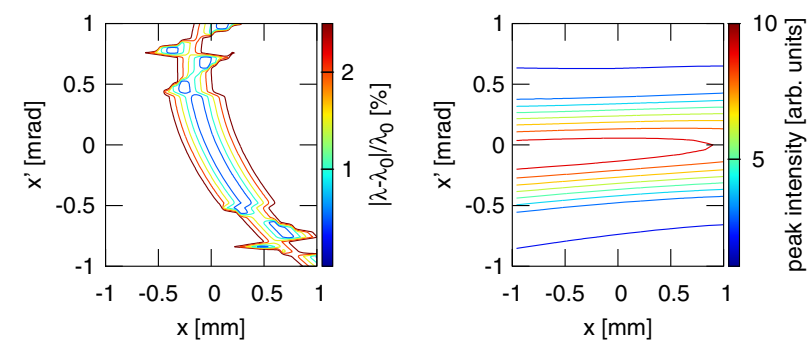

FIG. 13. Dynamic acceptance levels for $120 \mathrm{MeV}$ electrons entering the TGU in terms of the relative deviation of the mean wavelength of the emitted radiation from that emitted by the reference particle (left) and in terms of the peak intensity of the emitted radiation (right).

is large (note, however, that the geometric acceptance is not) and the coupling between $x$ - and $y$-phase space is weak, we disregard the vertical coordinate here and assume $y=0, y^{\prime}=0$. Figure 14 shows the boundaries of the phase space areas for three discrete electron energies $\left(E_{0} \pm 10 \%\right)$ to which the electron beam must be matched if a radiation bandwidth close to the natural bandwidth of $1 \%$ is demanded. In our example we assume that the bandwidth of the incoherently added total radiation spectrum can be estimated by a convolution of the distribution of wavelengths emitted by the particles contained in the acceptance area with the spectral function of a 100-period undulator. Under this assumption, for a bandwidth of the total radiation spectrum of $1.1 \%$ an acceptance level of $0.5 \%$ in the above defined sense is required. Additionally, the phase space boundaries for a radiation intensity level of $50 \%$ are shown in the figure.

We note that for all energies the acceptance area is in the order of $10^{-8} \mathrm{mrad}$, particularly admitting a rather large beam divergence. Due to its curved shape, however. the acceptance area is not fully exploitable by means of linear matching optics. In addition, the acceptance area is not symmetric in $x^{\prime}$, i.e. a small nonzero initial angle of the beam is favored.

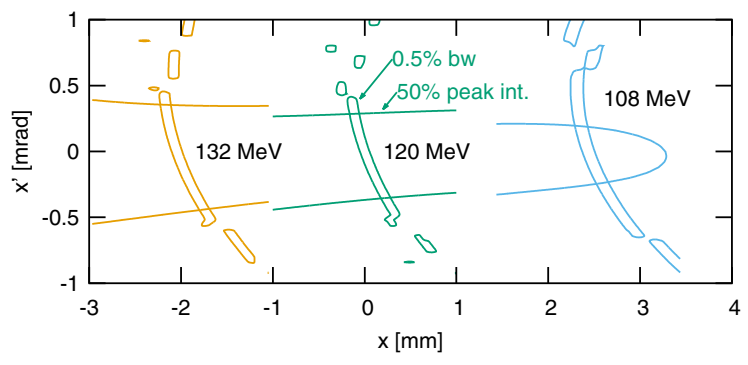

FIG. 14. Dynamic acceptance for maximum, central and minimum electron energy for an acceptance level of $0.5 \%$ relative deviation of the radiated wavelength (after convolution resulting in $1.1 \%$ bandwidth of the incoherently added spectrum) and $50 \%$ of the radiated peak intensity as compared to the wavelength and peak intensity radiated by the reference particle.

\section{TOWARDS A PROOF-OF-PRINCIPLE EXPERIMENT}

In the previous section we have considered general conditions one has to take into account in any experimental setup involving a transverse gradient undulator irrespective of the fact that we used a particular example case for our study. In this section we turn away from our idealized example case towards the example of the superconducting cylindric TGU realized at KIT $[13,17]$ and to be tested experimentally at the JETI laser in Jena.

The basic parameters of this SCTGU are the same as for our idealized example case except for the number of periods (see also Table II). The real SCTGU features 40 periods plus two $1 / 4,3 / 4$ matching periods as described above. The winding scheme for the superconducting wire requires a deviation from the ideal cylindric shape of the undulator coils: In order to accommodate the wire jumps, every second winding package is laid out as a racetrack coil. The contributions of the differently shaped winding packages add up to a nonvanishing horizontal magnetic stray field at the entrance and the exit of the SCTGU, resulting in a vertical deflection of the electron beam. Therefore a vertical steering of the beam is required, adjusting the vertical offset and angle under which the electrons enter the TGU.

With these two parameters optimized in addition to the spectral dispersion and the optical functions of the electron beam, we expect to achieve the radiation spectra shown in Fig. 15. The beam parameters and optical functions assumed in the calculation of these spectra are summarized in Table II. Again, the radiation emitted parallel in the forward direction as it would be collected by an energydispersive photon optics with a very small angular acceptance, and the radiation observed by an energy-dispersive

TABLE II. Parameters for a proof-of-principle experiment.

Electron beam

Beam energy

Average beam current

Energy spread (Gaussian)

Geometric emittances

Target vertical beta function

Vertical entrance angle

Target horizontal beta function

Target dispersion (parallel beam)

Target dispersion (point detector)

Undulator

Period length

Periods

Undulator strength

Transverse gradient
$E_{0}=120 \mathrm{MeV}$

$I=10 \mathrm{pC} \times 1 \mathrm{~Hz}$

$\sigma_{E}=0.05$

$\epsilon_{x, y}=10 \mathrm{nmrad}$

$\beta_{y}=0.7 \mathrm{~m}=\mathrm{const}$

$\theta_{y}=1.0 \mathrm{mrad}$

$\beta_{x}^{\text {waist }}=0.2 \mathrm{~m}$

$\eta_{0}=2.2 \times 10^{-2} \mathrm{~m}$

$\eta_{1}=1.9 \times 10^{-2} \mathrm{~m}$

$\eta_{0}=2.2 \times 10^{-2} \mathrm{~m}$

$\eta_{1}=8.4 \times 10^{-2} \mathrm{~m}$

$\eta_{2}=4.4 \times 10^{-1} \mathrm{~m}$

$\lambda_{\mathrm{u}}=10.5 \mathrm{~mm}$

$N_{\mathrm{u}}=40$

$K_{0}=1.07$

$\alpha_{K}=149.5 \mathrm{~m}^{-1}$ 


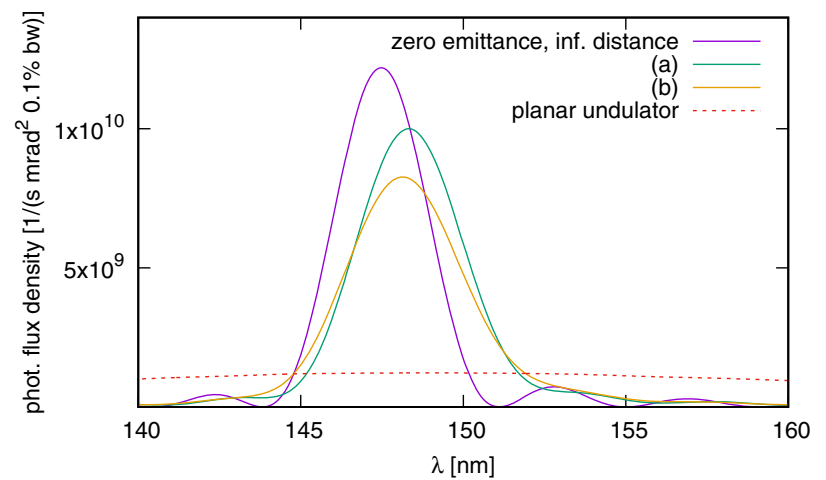

FIG. 15. Radiation spectra expected for the real SCTGU-P40 with finite beam emittance and observation at $2 \mathrm{~m}$ distance with (a) a photon optics for a parallel beam (zero angular acceptance) and (b) a point detector. The corresponding zero-emittance and planar undulator spectra in the forward direction (parallel beam) are added for comparison. For the undulator and beam parameters refer to Table II.

point detector are considered. The spectral dispersion of the electron beam for each case was adjusted according to the discussion in Sec. IV D. The respective linear and higher order dispersion terms are quoted in Table II.

Figure 15 shows also the TGU spectrum for a zeroemittance beam and the radiation spectrum of a planar undulator (for finite emittance) for comparison. No strong degradation of the spectral quality as compared to the zero emittance beam is observed which is a consequence of the increased natural bandwidth of the real undulator due to its reduced number of periods.

Clearly a significant enhancement is achieved in comparison with a planar undulator. As we consider an accelerator with $1 \mathrm{~Hz}$ repetition rate, the displayed spectra can be read as single-shot spectra of the forward or on-axis photon flux density, respectively. The numbers suggest that the SCTGU-P40 is very well suited for an experimental proof of principle with good prospects of success.

\section{CONCLUSIONS}

In this paper we have shown that the concept of generating monochromatic and intense undulator radiation with a large-energy-spread electron beam and a transverse gradient undulator is valid under realistic conditions. General limitations to the concept are imposed by a finite beam emittance, a finite length of the TGU and a finite observation distance, and the ponderomotive particle drift effect occurring due to the transverse field gradient.

Surprisingly, this ponderomotive drift does not significantly disturb the phase relation between the electron motion and the radiation emitted in forward direction. For an observer at infinite distance the transverse drift does not affect the bandwidth of the observed radiation spectrum. In practice, however, a compensation of the transverse particle drift is indispensable. The compensation scheme employed in our SCTGU is proved to work by the presented simulations.

A transverse gradient undulator can have a large energy acceptance. In our example we assume $\Delta E / E_{0}= \pm 10 \%$ equally distributed. We show, however, that a nonlinear spectral dispersion matching is required even in the case of an ideally linear transverse field amplitude decrease in the TGU, because already the deviation of the linear approximation for the dispersion from the exact solution leads to a significant shift of the radiated wavelength.

Nonlinear dispersion matching is also the key to effectively compensating the relativistic Doppler shift degrading the monochromaticity and intensity of the radiation observed in a point at finite distance. We show that even for observation distances in the order of a few meters the spectral bandwidth and intensity of the observed radiation can be recovered to a high extent by adjusting the spectral dispersion of the electron beam.

The most determining limitation to the conceptscaling, however, with the transverse field gradient-is imposed by the finite beam emittance. This limitation is evident already from a simple linear estimation derived from the undulator equation. Our calculations reveal that in fact the dynamic acceptance of a TGU is larger than expected from this estimation due to the rather large angular acceptance of the TGU. However, only a limited portion of the acceptance area in phase space is matchable by means of a linear beam transport optics. On the other hand, even with a not perfectly matched transverse phase space, a TGU will provide significantly enhanced radiation spectra than achievable with a planar undulator.

In conclusion, we show by combining all finite-scale effects discussed in this paper that the superconducting TGU recently realized at KIT is suited for a proof-of-principle experiment which we intend to perform in the near future.

Although the calculations presented in this paper refer to a particular idealized example case, our general findings apply to essentially any transverse gradient undulator.

As a final general conclusion, our simulations basically confirm the validity of naive estimations based on the undulator equation. In detail, however, they provide additional insight, especially regarding the TGU's dynamic acceptance and the required nonlinear dispersion matching. The effects observed in this respect will become increasingly important if the TGU length is scaled up e.g. in order to realize a TGU-FEL. Similarly realistic 3D particle dynamics simulations for the TGU-FEL case as the next step therefore appear highly desirable.

[1] T. I. Smith, J. M. J. Madey, L. R. Elias, and D. A. G. Deacon, Reducing the sensitivity of a free electron laser to electron energy, J. Appl. Phys. 50, 4580 (1979).

[2] G. Fuchert, A. Bernhard, S. Ehlers, P. Peiffer, R. Rossmanith, and T. Baumbach, A novel undulator concept 
for electron beams with a very large energy spread, Nucl. Instrum. Methods Phys. Res., Sect. A 672, 33 (2012).

[3] H.-P. Schlenvoigt, K. Haupt, A. Debus, F. Budde, O. Jäckel, S. Pfotenhauer, H. Schwoerer, E. Rohwer, J. Gallacher, E. Brunetti, R. Shanks, S. Wiggins, and D. Jaroszynski, A compact sychrotron radiation source driven by a laser plasma wakefield accelerator, Nat. Phys. 4, 130 (2008).

[4] Z. Huang, Y. Ding, and C. B. Schroeder, Compact X-ray Free-Electron-Laser from a Laser-Plasma Accelerator Using a Transverse-Gradient Undulator, Phys. Rev. Lett. 109, 204801 (2012).

[5] Y. Ding, P. Baxevanis, Y. Cai, Z. Huang, and R. Ruth, in Proceedings of the International Particle Accelerator Conference IPAC2013, Shanghai, China, 2013 (JACoW, Geneva, Switzerland, 2013), WEPWA075.

[6] T. Chanwattana, R. Bartolini, and A. Seryi, in Proceedings of the International Particle Accelerator Conference IPAC2014, Dresden, Germany, 2014 (JACoW, Geneva, Switzerland, 2014), THPRO032.

[7] F. Ciocci, G. Dattoli, and E. Sabia, Transverse Gradient Undulators and FEL operating with large energy spread, Opt. Commun. 356, 582 (2015).

[8] A. Bernhard, E. Burkard, V. A. Rodriguez, C. Widmann, and A.-S. Müller, in Proceedings of the International Particle Accelerator Conference IPAC2015, Richmond, Virginia, USA, 2015 (JACoW, Geneva, Switzerland, 2015), TUPWA039.

[9] P. Baxevanis, Y. Ding, Z. Huang, and R. Ruth, 3D theory of a high-gain free-electron laser based on a transverse gradient undulator, Phys. Rev. ST Accel. Beams 17, 020701 (2014).
[10] G. Fuchert, A. Bernhard, S. Ehlers, P. Peiffer, D. Wollmann, T. Baumbach, and R. Rossmanith, in Proceedings of the 31st International Free Electron Laser Conference (FEL 09), Liverpool, UK (STFC Daresbury Laboratory, Warrington, 2009).

[11] R. P. Walker, Phase errors and their effect on undulator radiation properties, Phys. Rev. ST Accel. Beams 16, 010704 (2013).

[12] C. Widmann, V. A. Rodriguez, A. Bernhard, N. Braun, B. Härer, O. Jäckel, M. Nicolai, P. Peiffer, M. Reuter, T. Rinck, R. Rossmanith, A. Sävert, M. Scheer, W. Walter, T. Baumbach, and M. Kaluza, in Proceedings of the International Particle Accelerator Conference IPAC2013, Shanghai, China, 2013 (JACoW, Geneva, Switzerland, 2013), TUPWO013.

[13] V. A. Rodriguez, A. Bernhard, A. Keilmann, P. Peiffer, R. Rossmanith, C. Widmann, T. Baumbach, M. Nicolai, and M. C. Kaluza, Development of a non-planar superconducting undulator for the JETI-laser-wakefield accelerator, IEEE Trans. Appl. Supercond. 23, 4101505 (2013).

[14] V. A. Rodríguez, Ph.D. thesis, Karlsruhe Institute of Technology, 2015.

[15] M. Scheer, in Proceedings of the 3rd International Particle Accelerator Conference, New Orleans, Louisiana, USA, 2012 (IEEE, Piscataway, NJ, 2012), TUACC2.

[16] OpERA-3D, Cobham Technical Services, Vector Fields Software, 2014.

[17] V. A. Rodriguez, A. Bernhard, A. Grau, P. Peiffer, R. Rossmanith, M. Weber, C. Widmann, A. Will, M. Kaluza, M. Nicolai, A. Sävert, and M. Reuter, in Proceedings of the International Particle Accelerator Conference IPAC2014, Dresden, Germany, 2014 (JACoW, Geneva, Switzerland, 2014). 